\title{
A Granular Consensus Model Based on Intuitionistic Reciprocal Preference Relations and Minimum Adjustment for Multi-Criteria Group Decision Making
}

\author{
Francisco Javier Cabrerizo $^{a}$ and José Ramón Trillo ${ }^{a}$ and Juan Antonio Morente-Molinera ${ }^{a}$ \\ and Sergio Alonso ${ }^{a}$ and Enrique Herrera-Viedma ${ }^{a}$ \\ ${ }^{a}$ Department of Computer Science and Artificial Intelligence, Andalusian Research Institute in Data Science and \\ Computational Intelligence (DaSCI), University of Granada, 18071, Granada, Spain \\ cabrerizo@decsai.ugr.es, jrtrillo@ugr.es, jamoren@decsai.ugr.es \\ zerjioi@ugr.es, viedma@decsai.ugr.es
}

\begin{abstract}
When a group of individuals try to collectively make a decision, it is important that all of them accept the decision adopted. It means, to improve consensus, some adjustments could be inevitably performed to the initial assessments given by the individuals. To do it, several models have been recently developed from the viewpoint of the granular computing paradigm. However, the models dealing with intuitionistic reciprocal preference relations do not consider that the modified assessments could be very different from the initial ones. The aim of this work is to develop a model based on the granular computing paradigm that tries to increase the consensus at the same time that tries to reduce the dissimilarity between the original assessments and the adjusted ones. In addition to it, this model is able to deal with multicriteria group decision making problems.
\end{abstract}

Keywords: Consensus, Granular computing, Intuitionistic reciprocal preference relations, Minimum adjustment, Multi-criteria group decision making.

\section{Introduction}

Group decision making, otherwise called collaborative decision making, characterizes a context in which a number of individuals collectively arrive at a choice from a collection of alternatives [26]. Instead of being attributable to a single member of the group, the decision is now attributable to all the individuals as all of them contribute to the outcome [6]. A decision made by an individual is often different from that made by a group. Due to it, group decision making is the most popular model to produce buy-in from other people, support creativity, and build consensus [20].
Consensus makes an effort to avoid "losers" and "winners", it needs the major part of a group of individuals approves the decision made, but the other part also agrees to it [16]. It means consensus is an iterative way of reaching agreement between the individuals of a group [13]. It ensures all individuals are attentively listened. In such a way, as a result of keeping in mind all ideas, concerns, and opinions, the group of individuals aims to make decisions that are useful for all.

To reach agreement, the members of the group must be prepared for changing their initial positions, i.e., every member of the group has to allow a specific level of adaptability in which her or his first position may be changed. Within the recent past, a number of models developed from the viewpoint of the granular computing have been proposed to deal with group decision making problems defined in fuzzy environments $[9,10,21,25]$. Granular computing introduces a logical framework utilizing concepts, such as information granularity or information granules, for managing knowledge gathered and handled at distinct levels of abstraction [24]. In particular, the concept of information granularity has played an important role in this kind of problems [23]. For instance, it has been used to estimate missing information [7] and to improve consistency related to the assessments given by the individuals [10]. Related to the consensus, the topic studied in this work, information granularity has been also used to support it. For instance, different models have been developed in the context of the analytic hierarchy process $[21,25]$ and in group decision making problems in which fuzzy preference relations $[9,11]$ and intuitionistic reciprocal preference relations [8] are used to model the assessments given by the members of the group.

Most models using the concept of information granularity that have been constructed to improve the consensus do not consider that the new assessments suggested could be to some extent different from the first assessments provided by the individuals. By the defi- 
nition of consensus, it is assumed that individuals accept to change their initial viewpoints. However, if the new assessments suggested are far from the first ones provided, individuals could not accept the new ones. For instance, let us suppose an individual thinks that a car is travelling at an average speed of 100 kilometers per hour. In this case, whether the adjusted average speed suggested by the model is equal to 95 kilometers per hour, this individual could accept it in order to contribute to the consensus. However, whether the adjusted average speed is equal to 50 kilometers per hour, which is very different from the first opinion provided by the individual, he or she could not accept it. Hence, the model must minimize the dissimilarity between the first assessment provided by the individual and the suggested one by reducing the adjustment $[9,32]$.

Another question that must be considered is that of taking into account a number of criteria to assess the alternatives. For instance, let us suppose we want to drink wine and we must choose between four brands of wines. With the aim of choosing the best wine, different criteria should be considered: color, appearance, body of wine, odor of wine, taste, and so on. However, most models using the concept of information granularity to improve the consensus assume that individuals assess the alternatives as a whole instead of considering different criteria to evaluate them.

This work aims to build a new granular consensus model for multi-criteria group decision making. First, assuming intuitionistic reciprocal preference relations [28] to model the assessments provided by the individuals of the group and being based on the concept of information granularity, this new model improves the consensus at the same time that reduces the dissimilarity between the original assessments and the suggested ones. Second, it is able to deal with decision making contexts in which a number of criteria are taken into account to assess the collection of alternatives.

The rest of this work is organized as follows. Section 2 recalls both the definition of a group decision making problem in a multi-criteria context and the definition of an intuitionistic reciprocal preference relation. The new granular consensus model proposed in this work is fully described in Section 3. An experimental study is carried out in Section 4. Its results are also analyzed in this section. Finally, Section 5 concludes the work and points out several future research directions.

\section{Preliminaries}

We recall the group decision making problems defined in multi-criteria contexts and the intuitionistic reciprocal preference relations in this section.

\subsection{Multi-criteria group decision making}

According to the individuals' assessments, the most appropriate alternative is obtained between a collection of possible options by producing a ranking of them [25]. Instead of considering an only one criterion, it is frequent that individuals have multiple criteria in mind when assessing the alternatives [18]. Then, we face a multi-criteria group decision making problem, which is characterized by a finite collection of alternatives, $A=\left\{a_{1}, a_{2}, \ldots, a_{n}\right\}$; a finite group of individuals, $I=\left\{i_{1}, i_{2}, \ldots, i_{z}\right\}$; a finite collection of criteria used to assess the alternatives, $C=\left\{c_{1}, c_{2}, \ldots, c_{q}\right\}$; and every criterion $c_{l} \in C$ has associated a weight of importance $w_{l} \in[0,1]$. Usually, they are normalized: $\sum_{l=1}^{q} w_{l}=1$.

The individuals' assessments on the alternatives usually represent the preference degree of one alternative over other one for a particular criterion or the degree up to which an alternative satisfies a given criterion. Any case, a particular representation domain must be chosen to characterize the assessments [17]. In particular, fuzzy set theory has been used in the resolution of decision making processes as they are cognitive processes in which participate individuals (humans) [4]. Actually, fuzzy set theory and its extensions have demonstrated to be helpful in representing decision information pervaded with human uncertainty [5].

\subsection{Intuitionistic reciprocal preference relations}

Fuzzy set theory assumes the complement of a fuzzy set $B$ has a membership function defined exclusively in regards to the membership function $\mu_{B}(y)$ as $1-\mu_{B}(y)$ [31]. It means adding the membership degree of every element $y$ of a universal set $Y$ to a fuzzy set $B$ and its complement $B^{c}$ is equal to 1 in this theory. However, the Atanassov's intuitionistic fuzzy set theory [1, 2, 3], one of the extensions of the fuzzy set theory, relaxes this fundamental assumption. Concretely, it assumes the referred sum rests on the condition of being equal or less than 1. It means the membership degree of every element $y$ of a universal set $Y$ to the complement fuzzy set of $B$ is equal or smaller than $1-\mu_{B}(y)$.

Definition 1 [1] "Let $Y$ be a non-empty universe. An Atanassov's intuitionistic fuzzy set (AIFS) $B$ on $Y$ is defined as $B=\left\{\left(y, \mu_{B}(y), v_{B}(y)\right) \mid y \in Y\right\}$ with $\mu_{B}(y) \in$ $[0,1]$ being the membership degree of the element $y \in Y$ in $B$ and $v_{B}(y) \in[0,1]$ being the non-membership degree of the element $y \in Y$ in $B$, subject to the constraint $0 \leq \mu_{B}(y)+v_{B}(y) \leq 1$.”

Related to every element $y \in Y$ in the studied set $B$, a hesitancy index is characterized as $\pi_{B}(y)=1-\mu_{B}(y)-$ $v_{B}(y)$. This index determines an individual's hesita- 
tion measure to give a numerical value to $\mu_{B}(y)$ and $v_{B}(y)$. Its advantage is to allow another freedom degree, which can model hesitancy of individuals concerning the inclusion and exclusion of an element to a given set [29]. It gives us an additional possibility of representing imperfect knowledge what leads to describing many real problems in a more adequate way.

Whether an AIFS is used to represent the assessment provided by an individual and this assessment is interpreted as the preference degree of one alternative over other one, it leads to pairwise comparison assessments between the available alternatives. In particular, it leads to intuitionistic reciprocal preference relations.

Definition 2 [28] "An intuitionistic reciprocal preference relation $R$ on the set $A$ is represented by a matrix $R=\left(r_{i j}\right)_{n \times n} \subset A \times A$ with $r_{i j}=$ $\left\langle\left(a_{i}, a_{j}\right), \mu_{R}\left(a_{i}, a_{j}\right), v_{R}\left(a_{i}, a_{j}\right)\right\rangle \forall i, j=1,2, \ldots, n$. For convenience, let $r_{i j}=\left\langle\mu_{i j}, v_{i j}\right\rangle \forall i, j=1,2, \ldots, n$, where $r_{i j}$ is an intuitionistic fuzzy value composed by the certainty degree $\mu_{i j}$ to which $a_{i}$ is preferred to $a_{j}$ and the certainty degree $v_{i j}$ to which $a_{i}$ is non-preferred to $a_{j}$, and $\pi_{i j}=1-\mu_{i j}-v_{i j}$ is interpreted as the uncertainty degree to which $a_{i}$ is preferred to $a_{j}$. Furthermore, $\mu_{i j}$ and $v_{i j}$ satisfy the following conditions:

$$
\begin{aligned}
0 \leq \mu_{i j}+v_{i j} \leq 1 & \forall i, j=1,2, \ldots, n \\
\mu_{i i}=v_{i i}=0.5 & \forall i=1,2, \ldots, n \\
\mu_{j i}=v_{i j} & \forall i, j=1,2, \ldots, n ”
\end{aligned}
$$

\section{Granular consensus model based on minimum adjustment}

This section elaborates on the granular consensus model for multi-criteria group decision making based on intuitionistic reciprocal preference relations and minimum adjustment that we propose in this work. This new model, which is based on the concept of information granularity, aims to increase the consensus among the individuals at the same time that reduces the dissimilarity between the original assessments given by them and the suggested ones. To do so, the consensus model is structured into two phases, which are elaborated on next.

\subsection{Gathering assessments}

The starting point of this model is a multi-criteria group decision making defined as in Section 2.1 and the use of intuitionistic reciprocal preference relations to characterize the individuals' assessments. In effect, in this first phase of the model, every individual $i_{h}$, $h \in\{1, \ldots, z\}$, provides an (individual) intuitionistic reciprocal preference relation $R^{h l}$ for each criterion $c_{l}$, $l \in\{1, \ldots, q\}$.

\subsection{Improvement of consensus with minimum adjustment}

Once the individuals have provided their assessments via intuitionistic reciprocal preference relations and prior to make the decision, generally made by means of a selection process [15], the consensus among the individuals must be measured. The idea is that whether the measurement obtained is not enough (a threshold value is generally established [11]) the group is required to discuss again. This usually leads to the modification of the initial assessments to bring positions closer. To model it, the concept of information granularity serves as a suitable way of allowing the flexibility that is needed [23]. Concretely, it helps transforming the entries of the intuitionistic reciprocal preference relations, which are composed of two numerical values representing an AIFS, into information granules of higher abstraction level [22], leading to granular intuitionistic reciprocal preference relations $[8,10]$.

The notation $\mathbf{G}(R)$ is commonly used to call attention to the use of a granular version of an intuitionistic reciprocal preference relation $R$. Concretely, and similar like other proposals $[11,21,25]$, we consider the information granulation framework assumed in this model is based on intervals. It stands for $\mathbf{G}(R)=\mathbf{I}(R)$, being $\mathbf{I}(\cdot)$ a family of intervals. It brings out the concept of information granularity plays a notable role as synonym for the flexibility inserted into the components of the intuitionistic reciprocal preference relations. That is, the length of the intervals, which are characterized by an information granularity level $\alpha$, may be utilized to improve the consensus.

To sum up, we aim to improve the consensus by adjusting the assessments provided by the individuals, which are in the form of AIFSs, within the limits that the information granularity level $\alpha$ allows. In addition, we aim to reduce the dissimilarity between the assessments provided by the individuals and the adjusted ones. This is done via the particle swarm optimization (PSO) algorithm [19], which has been successfully applied in similar problems [10, 22, 23].

\subsubsection{Algorithm}

PSO starts with a swarm consisting of a number of particles depicted as positions in a search-space of $d$ dimensions, i.e., the particles represent potential solutions of the optimization task. By moving to a new position based on their previous positions and a new velocity, the particles try to discover, in a iterative way, a solution that optimizes a fitness function $f$.

In this work, we assume the generic form of this algorithm (refer to [27] for a number of variants, which 
could be also used here). Considering that every particle $k$ is composed of three $d$-dimensional vectors, $\mathbf{v}_{k}=\left(v_{k, 1}, v_{k, 2}, \ldots, v_{k, d}\right), \mathbf{x}_{k}=\left(x_{k, 1}, x_{k, 2}, \ldots, x_{k, d}\right)$ and $\mathbf{x b}_{k}=\left(x b_{k, 1}, x b_{k, 2}, \ldots, x b_{k, d}\right)$, representing its velocity, current position and best position achieved so far, respectively, the following expressions control the next velocity and position of the particle [19]:

$$
\begin{aligned}
v_{k, h}(t+1)= & \omega \cdot v_{k, h}(t)+c_{1} \cdot r_{h} \cdot\left(x b_{k, h}(t)-x_{k, h}(t)\right) \\
& +c_{2} \cdot s_{h} \cdot\left(x g_{h}(t)-x_{k, h}(t)\right) \\
x_{k, h}(t+1)= & x_{k, h}(t)+v_{k, h}(t+1)
\end{aligned}
$$

being $t$ the current iteration and $h$ the dimension of the particle $k ; \omega$, called inertia weight, serves to scale the current velocity (a small value means local exploration and a high value means global exploration); $c_{1}$ and $c_{2}$ denote two acceleration coefficients modeling the step size the particle takes in the direction of its best position and in the direction of the best global position, respectively; $\mathbf{r}=\left(r_{1}, r_{2}, \ldots, r_{d}\right)$ and $\mathbf{s}=\left(s_{1}, s_{2}, \ldots, s_{d}\right)$ are two vectors of values from two random sequences in $[0,1]$; and $\mathbf{x g}=\left(x g_{1}, x g_{2}, \ldots, x g_{d}\right)$ is a vector characterizing the global best position achieved by a particle of the swarm.

\subsubsection{Particle's representation}

Based on the distinguishing features of the multicriteria group decision making problem modeled, every particle is characterized by a vector whose components belong to the closed interval $[0,1]$. Concretely, whether the problem is set up with $q$ criteria, $n$ alternatives and $z$ individuals, every particle consists in a vector of $q \cdot(n-1) \cdot n \cdot z$ dimensions.

Let $r_{i j}=\left\langle\mu_{i j}, v_{i j}\right\rangle$ be an element of an intuitionistic reciprocal preference relation $R$. Whether $\alpha$ is the information granularity level established, the components, $\mu_{i j}$ and $v_{i j}$, can take values within the intervals $\left[\mathrm{L} \mu_{i j}, \mathrm{U} \mu_{i j}\right]$ and $\left[\mathrm{L} v_{i j}, \mathrm{U} v_{i j}\right]$, respectively, in $\mathbf{I}(R)$ :

$$
\begin{aligned}
& {\left[\mathrm{L} \mu_{i j}, \mathrm{U} \mu_{i j}\right]=\left[\max \left(0, \mu_{i j}-\frac{\alpha}{2}\right), \min \left(1, \mu_{i j}+\frac{\alpha}{2}\right)\right]} \\
& {\left[\mathrm{L} v_{i j}, \mathrm{U} v_{i j}\right]=\left[\max \left(0, v_{i j}-\frac{\alpha}{2}\right), \min \left(1, v_{i j}+\frac{\alpha}{2}\right)\right]}
\end{aligned}
$$

For illustrative purposes, let $r_{24}$ and $\alpha$ be $\langle 0.3,0.6\rangle$ and 0.3 , respectively. Let $x_{k, h}=0.4$ and $x_{k, h+1}=0.2$ be the corresponding components of the particle $k$. According to (6) and (7), the corresponding intervals to $\mu_{24}$ and $v_{24}$ are $[0.15,0.45]$ and $[0.45,0.75]$, respectively. Finally, using (8) and (9), we get that the adjusted values, $\bar{\mu}_{24}$ and $\bar{v}_{24}$, of $r_{24}$ are 0.27 and 0.51 , respectively, i.e., $\bar{r}_{24}=\langle 0.27,0.51\rangle$.

$$
\begin{aligned}
& \bar{\mu}_{i j}=\mathrm{L} \mu_{i j}+\left(\mathrm{U} \mu_{i j}-\mathrm{L} \mu_{i j}\right) \cdot x_{k, h} \\
& \bar{v}_{i j}=\mathrm{L} v_{i j}+\left(\mathrm{U} v_{i j}-\mathrm{L} v_{i j}\right) \cdot x_{k, h+1}
\end{aligned}
$$

\subsubsection{Fitness function}

The particle's objective is to optimize the return value of the fitness function $f$ at its position. In this work, by adjusting the initial assessments communicated by the individuals, first, we want to maximize the consensus achieved and, second, we want to decrease the dissimilarity between the adjusted assessments and the initial ones. According to these considerations:

$$
f=\gamma \cdot c r+(1-\gamma) \cdot(1-d s)
$$

being $\gamma \in[0,1]$ a parameter establishing a tradeoff between the consensus reached, $c r$, and the dissimilarity, $d s$, between the intuitionistic reciprocal preference relations provided by the individuals, $R^{h l}$, and the adjusted ones, $\bar{R}^{h l}, h \in\{1, \ldots, z\}$ and $l \in\{1, \ldots, q\}$.

According to (10), we need to compute two values, i.e. $c r$ and $d s$, in order to get the value of the fitness function $f$. To compute $c r$, we introduce a new approach based on the three levels of an intuitionistic reciprocal preference relation. Similar to other methodologies computing the consensus achieved by the members of a group, it is based on the concept of coincidence [14]. This new approach presents the following steps:

- Computation of a matrix for every pair of individuals, $i_{g}$ and $i_{h}$, and every criterion, $c_{l}$, denoted as $S M=\left(s m_{i j}^{g h l}\right)$, that characterizes the similarity between the assessments communicated by these pair of individuals according to that criterion:

$$
s m_{i j}^{g h l}=1-d\left(r_{i j}^{g l}, r_{i j}^{h l}\right)
$$

being $d$ a metric that determines the distance between two AIFSs [30].

- Computation of a matrix for every criterion, $c_{l}$, denoted as $C M^{l}=\left(\mathrm{cm}_{i j}^{l}\right)$, that characterizes the consensus achieved by the group on that criterion:

$$
c m_{i j}^{l}=\frac{2}{z \cdot(z-1)} \sum_{g=1}^{z-1} \sum_{h=g+1}^{z} s m_{i j}^{g h l}
$$

- Computation, for every matrix $C M^{l}$, of three consensus measures: a measure of consensus, $c p_{i j}^{l}$, associated with a pair of alternatives, $a_{i}$ and $a_{j}$, a measure of consensus, $c a_{i}^{l}$, associated with an alternative, $a_{i}$, and a measure of global consensus, $c r^{l}$. These consensus measures are computed as:

$$
\begin{aligned}
c p_{i j}^{l} & =c m_{i j}^{l} \\
c a_{i}^{l} & =\frac{1}{2 \cdot(n-1)} \sum_{j=1 ; j \neq i}^{n}\left(c p_{i j}^{l}+c p_{j i}^{l}\right) \\
c r^{l} & =\frac{1}{n} \sum_{i=1}^{n} c a_{i}^{l}
\end{aligned}
$$


- Computation of $c r$ via the weighted average of the measures of global consensus associated with the criteria:

$$
c r=\sum_{l=1}^{q} w_{l} \cdot c r^{l}
$$

To compute $d s$, we need to obtain the dissimilarity between the first assessments provided by the individuals and the adjusted ones. It is done as:

- Computation of a dissimilarity index for every individual, $i_{h}$, and every criterion, $c_{l}$, denoted as $d s^{h l}$, that characterizes the dissimilarity between the assessments communicated by that individual and the suggested ones on that criterion:

$$
d s^{h l}=\frac{1}{n \cdot(n-1)} \sum_{i=1}^{n} \sum_{j=1 ; j \neq i}^{n} d\left(r_{i j}^{h l}, \bar{r}_{i j}^{h l}\right)
$$

being $d$ a metric that determines the distance between two AIFSs [30].

- Computation of a dissimilarity index for every criterion, $c_{l}$, denoted as $d s^{l}$, that characterizes the dissimilarity between the assessments communicated by the group of individuals and the suggested ones on that criterion:

$$
d s^{l}=\frac{1}{z} \sum_{h=1}^{z} d s^{h l}
$$

- Computation of $d s$ via the weighted average of the dissimilarity indexes related to the criteria:

$$
d s=\sum_{l=1}^{q} w_{l} \cdot d s^{l}
$$

\section{Experimental study}

This section carries out an experimental study illustrating this new granular consensus model and analyzing its results. Let $I=\left\{i_{1}, i_{2}, i_{3}, i_{4}\right\}$ be four estate agents evaluating four houses for sale, $A=\left\{a_{1}, a_{2}, a_{3}, a_{4}\right\}$, according to these three criteria, $C=\left\{c_{1}=\right.$ distance to center, $c_{2}=$ age, $c_{3}=$ number of rooms $\}$, being their importance weights: $w_{1}=0.3, w_{2}=0.2$ and $w_{3}=0.5$.

The first estate agent gives these intuitionistic reciprocal preference relations:

$$
\begin{aligned}
& R^{11}=\left[\begin{array}{llll}
\langle 0.50,0.50\rangle & \langle 0.70,0.30\rangle & \langle 0.60,0.40\rangle & \langle 0.30,0.70\rangle \\
\langle 0.30,0.70\rangle & \langle 0.50,0.50\rangle & \langle 0.50,0.40\rangle & \langle 0.30,0.70\rangle \\
\langle 0.40,0.60\rangle & \langle 0.40,0.50\rangle & \langle 0.50,0.50\rangle & \langle 0.60,0.40\rangle \\
\langle 0.70,0.30\rangle & \langle 0.70,0.30\rangle & \langle 0.40,0.60\rangle & \langle 0.50,0.50\rangle
\end{array}\right] \\
& R^{12}=\left[\begin{array}{llll}
\langle 0.50,0.50\rangle & \langle 0.70,0.20\rangle & \langle 0.70,0.30\rangle & \langle 0.20,0.70\rangle \\
\langle 0.20,0.70\rangle & \langle 0.50,0.50\rangle & \langle 0.70,0.20\rangle & \langle 0.20,0.70\rangle \\
\langle 0.30,0.70\rangle & \langle 0.20,0.70\rangle & \langle 0.50,0.50\rangle & \langle 0.40,0.60\rangle \\
\langle 0.70,0.20\rangle & \langle 0.70,0.20\rangle & \langle 0.60,0.40\rangle & \langle 0.50,0.50\rangle
\end{array}\right]
\end{aligned}
$$

$$
R^{13}=\left[\begin{array}{llll}
\langle 0.50,0.50\rangle & \langle 0.50,0.50\rangle & \langle 0.50,0.50\rangle & \langle 0.50,0.50\rangle \\
\langle 0.50,0.50\rangle & \langle 0.50,0.50\rangle & \langle 0.30,0.70\rangle & \langle 0.60,0.30\rangle \\
\langle 0.50,0.50\rangle & \langle 0.70,0.30\rangle & \langle 0.50,0.50\rangle & \langle 0.20,0.70\rangle \\
\langle 0.50,0.50\rangle & \langle 0.30,0.60\rangle & \langle 0.70,0.20\rangle & \langle 0.50,0.50\rangle
\end{array}\right]
$$

The second estate agent gives these intuitionistic reciprocal preference relations:

$$
\begin{aligned}
& R^{21}=\left[\begin{array}{llll}
\langle 0.50,0.50\rangle & \langle 0.20,0.70\rangle & \langle 0.70,0.30\rangle & \langle 0.60,0.30\rangle \\
\langle 0.70,0.20\rangle & \langle 0.50,0.50\rangle & \langle 0.90,0.10\rangle & \langle 0.30,0.60\rangle \\
\langle 0.30,0.70\rangle & \langle 0.10,0.90\rangle & \langle 0.50,0.50\rangle & \langle 0.60,0.40\rangle \\
\langle 0.30,0.60\rangle & \langle 0.60,0.30\rangle & \langle 0.40,0.60\rangle & \langle 0.50,0.50\rangle
\end{array}\right] \\
& [0.50,0.50\rangle\langle 0.50,0.50\rangle\langle 0.30,0.70\rangle\langle 0.50,0.50\rangle] \\
& R^{22}=\langle 0.50,0.50\rangle\langle 0.50,0.50\rangle\langle 0.80,0.10\rangle\langle 0.70,0.30\rangle \\
& \langle 0.70,0.30\rangle\langle 0.10,0.80\rangle\langle 0.50,0.50\rangle\langle 0.20,0.70\rangle \\
& \langle 0.50,0.50\rangle\langle 0.30,0.70\rangle\langle 0.70,0.20\rangle\langle 0.50,0.50\rangle \\
& R^{23}=\left[\begin{array}{llll}
\langle 0.50,0.50\rangle & \langle 0.20,0.80\rangle & \langle 0.70,0.20\rangle & \langle 0.60,0.40\rangle \\
\langle 0.80,0.20\rangle & \langle 0.50,0.50\rangle & \langle 0.70,0.20\rangle & \langle 0.70,0.30\rangle \\
\langle 0.20,0.70\rangle & \langle 0.20,0.70\rangle & \langle 0.50,0.50\rangle & \langle 0.60,0.40\rangle \\
\langle 0.40,0.60\rangle & \langle 0.30,0.70\rangle & \langle 0.40,0.60\rangle & \langle 0.50,0.50\rangle
\end{array}\right]
\end{aligned}
$$

The third estate agent gives these intuitionistic reciprocal preference relations:

$$
\begin{aligned}
& R^{31}=\left[\begin{array}{llll}
\langle 0.50,0.50\rangle & \langle 0.70,0.20\rangle & \langle 0.60,0.40\rangle & \langle 0.30,0.70\rangle \\
\langle 0.20,0.70\rangle & \langle 0.50,0.50\rangle & \langle 0.60,0.30\rangle & \langle 0.80,0.10\rangle \\
\langle 0.40,0.60\rangle & \langle 0.30,0.60\rangle & \langle 0.50,0.50\rangle & \langle 0.90,0.10\rangle \\
\langle 0.70,0.30\rangle & \langle 0.10,0.80\rangle & \langle 0.10,0.90\rangle & \langle 0.50,0.50\rangle
\end{array}\right] \\
& R^{32}=\left[\begin{array}{llll}
\langle 0.50,0.50\rangle & \langle 0.40,0.60\rangle & \langle 0.40,0.60\rangle & \langle 0.20,0.80\rangle \\
\langle 0.60,0.40\rangle & \langle 0.50,0.50\rangle & \langle 0.50,0.50\rangle & \langle 0.80,0.20\rangle \\
\langle 0.60,0.40\rangle & \langle 0.50,0.50\rangle & \langle 0.50,0.50\rangle & \langle 0.20,0.70\rangle \\
\langle 0.80,0.20\rangle & \langle 0.20,0.80\rangle & \langle 0.70,0.20\rangle & \langle 0.50,0.50\rangle
\end{array}\right] \\
& R^{33}=\left[\begin{array}{llll}
\langle 0.50,0.50\rangle & \langle 0.80,0.20\rangle & \langle 0.20,0.80\rangle & \langle 0.20,0.70\rangle \\
\langle 0.20,0.80\rangle & \langle 0.50,0.50\rangle & \langle 0.40,0.60\rangle & \langle 0.50,0.50\rangle \\
\langle 0.80,0.20\rangle & \langle 0.60,0.40\rangle & \langle 0.50,0.50\rangle & \langle 0.70,0.20\rangle \\
\langle 0.70,0.20\rangle & \langle 0.50,0.50\rangle & \langle 0.20,0.70\rangle & \langle 0.50,0.50\rangle
\end{array}\right]
\end{aligned}
$$

And the fourth estate agent gives these intuitionistic reciprocal preference relations:

$$
\begin{aligned}
& R^{41}=\left[\begin{array}{llll}
\langle 0.50,0.50\rangle & \langle 0.80,0.10\rangle & \langle 0.20,0.70\rangle & \langle 0.60,0.30\rangle \\
\langle 0.10,0.80\rangle & \langle 0.50,0.50\rangle & \langle 0.60,0.40\rangle & \langle 0.20,0.70\rangle \\
\langle 0.70,0.20\rangle & \langle 0.40,0.60\rangle & \langle 0.50,0.50\rangle & \langle 0.60,0.40\rangle \\
\langle 0.30,0.60\rangle & \langle 0.70,0.20\rangle & \langle 0.40,0.60\rangle & \langle 0.50,0.50\rangle
\end{array}\right] \\
& R^{42}=\left[\begin{array}{llll}
\langle 0.50,0.50\rangle & \langle 0.70,0.30\rangle & \langle 0.50,0.50\rangle & \langle 0.70,0.30\rangle \\
\langle 0.30,0.70\rangle & \langle 0.50,0.50\rangle & \langle 0.40,0.60\rangle & \langle 0.70,0.20\rangle \\
\langle 0.50,0.50\rangle & \langle 0.60,0.40\rangle & \langle 0.50,0.50\rangle & \langle 0.50,0.50\rangle \\
\langle 0.30,0.70\rangle & \langle 0.20,0.70\rangle & \langle 0.50,0.50\rangle & \langle 0.50,0.50\rangle
\end{array}\right] \\
& R^{43}=\left[\begin{array}{llll}
\langle 0.50,0.50\rangle & \langle 0.70,0.30\rangle & \langle 0.10,0.90\rangle & \langle 0.10,0.90\rangle \\
\langle 0.30,0.70\rangle & \langle 0.50,0.50\rangle & \langle 0.20,0.70\rangle & \langle 0.20,0.70\rangle \\
\langle 0.90,0.10\rangle & \langle 0.70,0.20\rangle & \langle 0.50,0.50\rangle & \langle 0.30,0.70\rangle \\
\langle 0.90,0.10\rangle & \langle 0.70,0.20\rangle & \langle 0.70,0.30\rangle & \langle 0.50,0.50\rangle
\end{array}\right]
\end{aligned}
$$

Considering these assessments, the consensus, $c r$, among the group of estate agents is 0.731 according to the approach presented in Section 3.2.3. This value has been obtained by using the Hamming distance between two AIFSs, $B_{1}$ and $B_{2}$, as $d$ in (11) and (17). However, other distance metrics of AIFSs could be used [30]. This distance is defined as [30]:

$$
\begin{aligned}
d\left(B_{1}, B_{2}\right)= & \frac{1}{2} \sum_{i=1}^{n}\left[\left|\mu_{B_{1}}\left(y_{i}\right)-\mu_{B_{2}}\left(y_{i}\right)\right|\right. \\
& \left.+\left|v_{B_{1}}\left(y_{i}\right)-v_{B_{2}}\left(y_{i}\right)\right|\right]
\end{aligned}
$$


If the consensus achieved must be greater than 0.75 before selecting the best house for sale, it means the estate agents must modify their assessments to increase the consensus and, here, our proposal comes into play.

Before showing the results generated by our proposal, we note the values assigned to the parameters of the PSO. Concretely, $c_{1}$ and $c_{2}$ were set to $2, \omega$ were set to 0.2 , the swarm consisted of 100 particles, and the number of iterations carried out were 300 . These values were assigned because of an intense experimentation. However, depending on the number of individuals, criteria and alternatives, these values can vary.

The results provided by our proposal for chosen values of $\alpha$ and $\gamma=0.5$ are shown in Table 1 . It can be observed that a higher value of $\alpha$ allows a higher flexibility. Consequently, the consensus achieved, $c r$, is greater. As an example, a consensus equal to 0.795 is reached whether $\alpha=0.4$, however, a greater value is obtained, $c r=0.922$, whether a higher granularity level is assumed, $\alpha=1.0$, in this case. On the contrary, a higher flexibility means a greater probability of producing adjusted assessments that could be very different from those initially given by the individuals. As an example, a dissimilarity equal to 0.077 is achieved whether $\alpha=0.4$, however, a greater value is reached, $d s=0.152$, whether a higher granularity level is considered, $\alpha=1.0$, in this case.

To analyze the impact of the parameter $\gamma$ in the composite fitness function $f$, Table 2 shows the results achieved by our proposal for chosen values of $\gamma$ and $\alpha=0.5$. These results reflect that a higher consensus is reached whether $\gamma=1.0$, which is logical as, in such a case, the optimization only concerns the consensus. On the contrary, whether $\gamma$ takes values different from 1 , the fitness function $f$ incorporates the effect of the dissimilarity, $d s$, and, therefore, the consensus reached is lower because the optimization is not only focused on maximizing the consensus, $c r$. It means lower values of $\gamma$ imply a lower consensus, $c r$, and a lower dissimilarity, $d s$, because more importance is given to the dissimilarity between the assessment provided by the individuals and the adjusted ones.

\begin{tabular}{cccc}
\hline & $f$ & $c r$ & $d s$ \\
\hline$\alpha=0.2$ & 0.858 & 0.755 & 0.040 \\
$\alpha=0.4$ & 0.859 & 0.795 & 0.077 \\
$\alpha=0.6$ & 0.863 & 0.830 & 0.104 \\
$\alpha=0.8$ & 0.871 & 0.864 & 0.123 \\
$\alpha=1.0$ & 0.885 & 0.922 & 0.152
\end{tabular}

Table 1: Values of $f, c r$ and $d s$ for $\gamma=0.5$ and fixed values of $\alpha$.

\begin{tabular}{cccc}
\hline & $f$ & $c r$ & $d s$ \\
\hline$\gamma=0.0$ & 0.919 & 0.754 & 0.081 \\
$\gamma=0.2$ & 0.888 & 0.774 & 0.083 \\
$\gamma=0.4$ & 0.865 & 0.794 & 0.087 \\
$\gamma=0.6$ & 0.842 & 0.798 & 0.093 \\
$\gamma=0.8$ & 0.822 & 0.801 & 0.096 \\
$\gamma=1.0$ & 0.807 & 0.807 & 0.103 \\
\hline
\end{tabular}

Table 2: Values of $f, c r$ and $d s$ for $\alpha=0.5$ and fixed values of $\gamma$.

Finally, as an example of the assessments generated by the proposed granular consensus model, we show the adjusted intuitionistic reciprocal preference relations obtained when $\alpha=0.2$ and $\gamma=0.5$ :

\begin{tabular}{|c|c|c|c|c|}
\hline \multirow{4}{*}{$\bar{R}^{11}=$} & {$[\langle 0.50,0.50\rangle$} & $\langle 0.63,0.23\rangle$ & $\langle 0.51,0.33\rangle$ & $\langle 0.22,0.62\rangle$ \\
\hline & $\langle 0.23,0.63\rangle$ & $\langle 0.50,0.50\rangle$ & $\langle 0.43,0.32\rangle$ & $\langle 0.20,0.60\rangle$ \\
\hline & $\langle 0.33,0.51\rangle$ & $\langle 0.32,0.43\rangle$ & $\langle 0.50,0.50\rangle$ & $\langle 0.53,0.31\rangle$ \\
\hline & {$[\langle 0.62,0.22\rangle$} & $\langle 0.60,0.20\rangle$ & $\langle 0.31,0.53\rangle$ & $\langle 0.50,0.50\rangle$ \\
\hline \multirow{4}{*}{$\bar{R}^{12}=$} & {$[\langle 0.50,0.50\rangle$} & $\langle 0.61,0.14\rangle$ & $\langle 0.62,0.23\rangle$ & $\langle 0.12,0.62\rangle$ \\
\hline & $\langle 0.14,0.61\rangle$ & $\langle 0.50,0.50\rangle$ & $\langle 0.63,0.12\rangle$ & $\langle 0.13,0.63\rangle$ \\
\hline & $\langle 0.23,0.62\rangle$ & $\langle 0.12,0.63\rangle$ & $\langle 0.50,0.50\rangle$ & $\langle 0.32,0.52\rangle$ \\
\hline & {$[\langle 0.62,0.12\rangle$} & $\langle 0.63,0.13\rangle$ & $\langle 0.52,0.32\rangle$ & $\langle 0.50,0.50\rangle$ \\
\hline \multirow{4}{*}{$\bar{R}^{13}=$} & {$[\langle 0.50,0.50\rangle$} & $\langle 0.43,0.42\rangle$ & $\langle 0.41,0.40\rangle$ & $\langle 0.42,0.42\rangle$ \\
\hline & $\langle 0.42,0.43\rangle$ & $\langle 0.50,0.50\rangle$ & $\langle 0.28,0.63\rangle$ & $\langle 0.53,0.22\rangle$ \\
\hline & $\langle 0.40,0.41\rangle$ & $\langle 0.63,0.28\rangle$ & $\langle 0.50,0.50\rangle$ & $\langle 0.30,0.63\rangle$ \\
\hline & {$[\langle 0.42,0.42\rangle$} & $\langle 0.22,0.53\rangle$ & $\langle 0.63,0.30\rangle$ & $\langle 0.50,0.50\rangle$ \\
\hline \multirow{4}{*}{$\bar{R}^{21}=$} & {$[\langle 0.50,0.50\rangle$} & $\langle 0.12,0.62\rangle$ & $\langle 0.62,0.22\rangle$ & $\langle 0.50,0.22\rangle$ \\
\hline & $\langle 0.62,0.12\rangle$ & $\langle 0.50,0.50\rangle$ & $\langle 0.80,0.01\rangle$ & $\langle 0.23,0.50\rangle$ \\
\hline & $\langle 0.22,0.62\rangle$ & $\langle 0.01,0.80\rangle$ & $\langle 0.50,0.50\rangle$ & $\langle 0.52,0.33\rangle$ \\
\hline & {$[\langle 0.22,0.50\rangle$} & $\langle 0.50,0.23\rangle$ & $\langle 0.33,0.52\rangle$ & $\langle 0.50,0.50\rangle$ \\
\hline \multirow{4}{*}{$\bar{R}^{22}=$} & {$[\langle 0.50,0.50\rangle$} & $\langle 0.42,0.42\rangle$ & $\langle 0.21,0.61\rangle$ & $\langle 0.42,0.42\rangle$ \\
\hline & $\langle 0.42,0.42\rangle$ & $\langle 0.50,0.50\rangle$ & $\langle 0.73,0.02\rangle$ & $\langle 0.63,0.22\rangle$ \\
\hline & $\langle 0.61,0.21\rangle$ & $\langle 0.02,0.73\rangle$ & $\langle 0.50,0.50\rangle$ & $\langle 0.11,0.60\rangle$ \\
\hline & {$[\langle 0.42,0.42\rangle$} & $\langle 0.22,0.63\rangle$ & $\langle 0.60,0.11\rangle$ & $\langle 0.50,0.50\rangle$ \\
\hline \multirow{4}{*}{$\bar{R}^{23}=$} & {$[\langle 0.50,0.50\rangle$} & $\langle 0.30,0.70\rangle$ & $\langle 0.60,0.30\rangle$ & $\langle 0.51,0.33\rangle$ \\
\hline & $\langle 0.70,0.30\rangle$ & $\langle 0.50,0.50\rangle$ & $\langle 0.60,0.12\rangle$ & $\langle 0.60,0.23\rangle$ \\
\hline & $\langle 0.30,0.60\rangle$ & $\langle 0.12,0.60\rangle$ & $\langle 0.50,0.50\rangle$ & $\langle 0.53,0.34\rangle$ \\
\hline & {$[\langle 0.33,0.51\rangle$} & $\langle 0.23,0.60\rangle$ & $\langle 0.34,0.53\rangle$ & $\langle 0.50,0.50\rangle$ \\
\hline \multirow{4}{*}{$\bar{R}^{31}=$} & {$[\langle 0.50,0.50\rangle$} & $\langle 0.62,0.13\rangle$ & $\langle 0.53,0.32\rangle$ & $\langle 0.22,0.62\rangle$ \\
\hline & $\langle 0.13,0.62\rangle$ & $\langle 0.50,0.50\rangle$ & $\langle 0.54,0.23\rangle$ & $\langle 0.70,0.01\rangle$ \\
\hline & $\langle 0.32,0.53\rangle$ & $\langle 0.23,0.54\rangle$ & $\langle 0.50,0.50\rangle$ & $\langle 0.80,0.04\rangle$ \\
\hline & {$[\langle 0.62,0.22\rangle$} & $\langle 0.01,0.70\rangle$ & $\langle 0.04,0.80\rangle$ & $\langle 0.50,0.50\rangle$ \\
\hline \multirow{4}{*}{$\bar{R}^{32}=$} & {$[\langle 0.50,0.50\rangle$} & $\langle 0.31,0.50\rangle$ & $\langle 0.31,0.53\rangle$ & $\langle 0.20,0.72\rangle$ \\
\hline & $\langle 0.50,0.31\rangle$ & $\langle 0.50,0.50\rangle$ & $\langle 0.42,0.40\rangle$ & $\langle 0.72,0.13\rangle$ \\
\hline & $\langle 0.53,0.31\rangle$ & $\langle 0.40,0.42\rangle$ & $\langle 0.50,0.50\rangle$ & $\langle 0.12,0.62\rangle$ \\
\hline & {$[\langle 0.72,0.20\rangle$} & $\langle 0.13,0.72\rangle$ & $\langle 0.62,0.12\rangle$ & $\langle 0.50,0.50\rangle$ \\
\hline \multirow{4}{*}{$\bar{R}^{33}=$} & {$[\langle 0.50,0.50\rangle$} & $\langle 0.73,0.13\rangle$ & $\langle 0.12,0.70\rangle$ & $\langle 0.14,0.63\rangle$ \\
\hline & $\langle 0.13,0.73\rangle$ & $\langle 0.50,0.50\rangle$ & $\langle 0.33,0.53\rangle$ & $\langle 0.41,0.42\rangle$ \\
\hline & $\langle 0.70,0.12\rangle$ & $\langle 0.53,0.33\rangle$ & $\langle 0.50,0.50\rangle$ & $\langle 0.60,0.14\rangle$ \\
\hline & {$[\langle 0.63,0.14\rangle$} & $\langle 0.42,0.41\rangle$ & $\langle 0.14,0.60\rangle$ & $\langle 0.50,0.50\rangle$ \\
\hline \multirow{4}{*}{$\bar{R}^{41}=$} & {$[\langle 0.50,0.50\rangle$} & $\langle 0.70,0.02\rangle$ & $\langle 0.13,0.60\rangle$ & $\langle 0.51,0.23\rangle$ \\
\hline & $\langle 0.02,0.70\rangle$ & $\langle 0.50,0.50\rangle$ & $\langle 0.50,0.30\rangle$ & $\langle 0.13,0.60\rangle$ \\
\hline & $\langle 0.60,0.13\rangle$ & $\langle 0.30,0.50\rangle$ & $\langle 0.50,0.50\rangle$ & $\langle 0.54,0.30\rangle$ \\
\hline & {$[\langle 0.23,0.51\rangle$} & $\langle 0.60,0.13\rangle$ & $\langle 0.30,0.54\rangle$ & $\langle 0.50,0.50\rangle$ \\
\hline \multirow{4}{*}{$\bar{R}^{42}=$} & {$[\langle 0.50,0.50\rangle$} & $\langle 0.62,0.21\rangle$ & $\langle 0.42,0.44\rangle$ & $\langle 0.62,0.23\rangle$ \\
\hline & $\langle 0.21,0.62\rangle$ & $\langle 0.50,0.50\rangle$ & $\langle 0.33,0.52\rangle$ & $\langle 0.62,0.23\rangle$ \\
\hline & $\langle 0.44,0.42\rangle$ & $\langle 0.52,0.33\rangle$ & $\langle 0.50,0.50\rangle$ & $\langle 0.40,0.44\rangle$ \\
\hline & {$[\langle 0.23,0.62\rangle$} & $\langle 0.23,0.62\rangle$ & $\langle 0.44,0.40\rangle$ & $\langle 0.50,0.50\rangle$ \\
\hline
\end{tabular}


$\bar{R}^{43}=\left[\begin{array}{llll}\langle 0.50,0.50\rangle & \langle 0.64,0.23\rangle & \langle 0.07,0.80\rangle & \langle 0.20,0.80\rangle \\ \langle 0.23,0.64\rangle & \langle 0.50,0.50\rangle & \langle 0.12,0.62\rangle & \langle 0.30,0.62\rangle \\ \langle 0.80,0.07\rangle & \langle 0.62,0.12\rangle & \langle 0.50,0.50\rangle & \langle 0.23,0.63\rangle \\ \langle 0.80,0.20\rangle & \langle 0.62,0.30\rangle & \langle 0.63,0.23\rangle & \langle 0.50,0.50\rangle\end{array}\right]$

Considering these adjusted assessments, the consensus, $c r$, among the group of estate agents is now 0.755 , which is higher than 0.75 (threshold value). Therefore, the best house for sale can be then selected by applying a selection process like those developed in [15].

\section{Conclusions and future work}

In this work, we have introduced a new consensus model based on the granular computing paradigm for multi-criteria group decision making problems in which the assessments given by the members of the group are modeled via intuitionistic reciprocal preference relations. In contrast to the existing granular models dealing with intuitionistic reciprocal preference relations, first, it can handle decision making scenarios where several criteria are considered to assess the alternatives, and, second, it improves the consensus at the same time that reduces the dissimilarity between the first assessments provided by the members of the group and the suggested ones. Consequently, it allows us to reach a notable balance between reduction of dissimilarity and improvement of consensus.

The research carried out in this work could be continued as follows:

- In decision making processes, coherence in pairwise comparisons is required to ensure consistent decisions [12]. However, pairwise comparison between the alternatives have traps, i.e., it is quite possible to obtain cycles, especially when many individuals consider many alternatives and many criteria. In consequence, as an additional component to the fitness function, the consistency associated with the assessments provided by individual members could be incorporated.

- The new model focuses on suggesting assessments as close as possible to the initial assessments provided by the group members. In addition to this, the number of assessments adjusted should be reduced. In consequence, the number of assessments that should be modified could be incorporated as component to the fitness function.

- The new model assumes that individuals can directly provide AIFSs. However, it is not always easy at all unless the individuals are really familiar with them. Therefore, automatic procedures transforming natural information from individuals into AIFSs should be used.

\section{Acknowledgement}

This work has been supported by the Spanish State Research Agency through the project PID2019103880RB-I00 / AEI / 10.13039/501100011033.

\section{References}

[1] K. T. Atanassov, Intuitionistic fuzzy sets, Fuzzy Sets and Systems 20 (1) (1986) 87-96.

[2] K. T. Atanassov, Intuitionistic Fuzzy Sets: Theory and Applications, Springer-Verlag, Berlin Heidelberg, 1999.

[3] K. T. Atanassov, On Intuitionistic Fuzzy Sets Theory, Springer-Verlag, Berlin Heidelberg, 2012.

[4] R. E. Bellman, L. A. Zadeh, Decision-making in a fuzzy environment, Management Science 17 (4) (1970) 141-164.

[5] H. Bustince, E. Barrenechea, M. Pagola, J. Fernández, Z. S. Xu, B. Bedregal, J. Montero, H. Hagras, F. Herrera, B. De Baets, A historical account of types of fuzzy sets and their relationships, IEEE Transactions on Fuzzy Systems 24 (1) (2016) 179-194.

[6] C. T. Butler, A. Rothstein, On Conflict and Consensus: A Handbook on Formal Consensus Decision Making, Food Not Bombs Publishing, Takoma Park, WA, 2006.

[7] F. J. Cabrerizo, R. Al-Hmouz, A. Morfeq, M. A. Martínez, W. Pedrycz, E. Herrera-Viedma, Estimating incomplete information in group decision making: A framework of granular computing, Applied Soft Computing 86 (2020) 105930.

[8] F. J. Cabrerizo, J. A. Morente-Molinera, S. Alonso, W. Pedrycz, E. Herrera-Viedma, Improving consensus in group decision making with intuitionistic reciprocal preference relations: A granular computing approach, in: Proc. 2018 IEEE International Conference on System, Man, and Cybernetics (SMC 2018), Miyazaki, Japan, 2018, pp. 1471-1476.

[9] F. J. Cabrerizo, J. A. Morente-Molinera, S. Alonso, W. Pedrycz, E. Herrera-Viedma, A granular consensus approach with minimum adjustment for multi-criteria group decision making, in: Proc. 2020 IEEE International Conference on System, Man, and Cybernetics (SMC 2020), Toronto, Canada, 2020, pp. 76-81. 
[10] F. J. Cabrerizo, I. J. Pérez, J. A. MorenteMolinera, S. Alonso, E. Herrera-Viedma, A granular computing based approach for improving the consistency of intuitionistic reciprocal preference relations, in: S. Shahbazova, J. Kacprzyk, V. Balas, V. Kreinovich (Eds.), Recent Developments and the New Direction in Soft-Computing Foundations and Applications, Vol. 393 of Studies in Fuzziness and Soft Computing, Springer, Cham, 2021, pp. 457-469.

[11] F. J. Cabrerizo, R. Ureña, W. Pedrycz, E. HerreraViedma, Building consensus in group decision making with an allocation of information granularity, Fuzzy Sets and Systems 255 (2014) 115127.

[12] J. L. García-Lapresta, J. Montero, Consistency in preference modeling, in: B. Bouchon-Meunier, G. Coletti, R. R. Yager (Eds.), Modern Information Processing: From Theroy to Applications, Elsevier, Amsterdam, 2006, pp. 87-97.

[13] P. Gelderloos, Consensus: A New Handbook for Grassroots Social, Political, and Environmental Groups, See Sharp Press, Tucson, AZ, 2006.

[14] F. Herrera, E. Herrera-Viedma, J. L. Verdegay, Linguistic measures based on fuzzy coincidence for reaching consensus in group decision making, International Journal of Approximate Reasoning 16 (3-4) (1997) 309-334.

[15] F. Herrera, E. Herrera-Viedma, J. L. Verdegay, Choice processes for non-homogeneous group decision making in linguistic setting, Fuzzy Sets and Systems 94 (3) (1998) 287-308.

[16] E. Herrera-Viedma, F. J. Cabrerizo, J. Kacprzyk, W. Pedrycz, A review of soft consensus models in a fuzzy environment, Information Fusion 17 (2014) 4-13.

[17] E. Herrera-Viedma, I. Palomares, C.-C. Li, F. J. Cabrerizo, Y. C. Dong, F. Chiclana, F. Herrera, Revisiting fuzzy and linguistic decision making: Scenarios and challenges for making wiser decisions in a better way, IEEE Transactions on Systems, Man, and Cybernetics: Systems 51 (1) (2021) 191-208.

[18] C.-L. Hwang, M.-J. Lin, Group Decision Making Under Multiple Criteria: Methods and Applications, Springer-Verlag, Berlin Heidelberg, 1987.

[19] J. Kennedy, R. C. Eberhart, Particle swarm optimization, in: Proc. IEEE International Conference on Neural Networks, Perth, Australia, 1995, pp. 1942-1948.
[20] J. R. Larson, In Search of Synergy in Small Group Performance, Psychology Press, New York, NY, 2010.

[21] F. Liu, Y. Wu, W. Pedrycz, A modified consensus model in group decision making with an allocation of information granularity, IEEE Transactions on Fuzzy Systems 5 (26) (2018) 31823187.

[22] S. Liu, W. Pedrycz, A. Gacek, Y. Dai, Development of information granules of higher type and their applications to granular models of time series, Engineering Applications of Artificial Intelligence 71 (2018) 60-72.

[23] W. Pedrycz, Allocation of information granularity in optimization and decision-making models: Towards building the foundations of Granular Computing, European Journal of Operational Research 232 (1) (2014) 137-145.

[24] W. Pedrycz, A. Skowron, V. Kreinovich, Handbook of Granular Computing, WileyInterscience, New York, NY, 2008.

[25] W. Pedrycz, M. Song, Analytich hierarchy process (AHP) in group decision making and its optimization with an allocation of information granularity, IEEE Transactions on Fuzzy Systems 19 (3) (2011) 527-539.

[26] T. L. Saaty, K. Peniwati, Group Decision Making: Drawing Out and Reconciling Differences, RWS Publications, Pittsburgh, PA, 2008.

[27] D. Wang, D. Tan, L. Liu, Particle swarm optimization algorithm: An overview, Soft Computing 22 (2018) 387-408.

[28] Z. S. Xu, Intuitionistic preference relations and their applications in group decision making, Information Sciences 177 (11) (2007) 2363-2379.

[29] Z. S. Xu, N. Zhao, Information fusion for intuitionistic fuzzy decision making: An overview, Information Fusion 28 (2016) 10-23.

[30] Y. Yang, F. Chiclana, Consistency of 2D and 3D distances of intuitionistic fuzzy sets, Expert Systems With Applications 39 (10) (2012) 86658670 .

[31] L. A. Zadeh, Fuzzy sets, Information and Control 8 (3) (1965) 338-353.

[32] B. Zhang, Y. C. Dong, Y. Xu, Multiple attribute consensus rule with minimum adjustments to support consensus reaching, Knowledge-Based Systems 67 (2014) 35-48. 